\title{
Structured Semidefinite Representation of Some Convex Sets
}

\author{
J. William Helton and Jiawang Nie \\ Department of Mathematics \\ University of California, San Diego \\ helton@math.ucsd.edu, njw@math.ucsd.edu
}

February 10, 2008

\begin{abstract}
Linear matrix Inequalities (LMIs) have had a major impact on control but formulating a problem as an LMI is an art. Recently there is the beginnings of a theory of which problems are in fact expressible as LMIs. For optimization purposes it can also be useful to have "lifts" which are expressible as LMIs. We show here that this is a much less restrictive condition and give methods for actually constructing lifts and their LMI representation.
\end{abstract}

\section{Introduction}

Recently, there is a lot of work Las01, ND05, NDS06, Par00, ParStu03, in solving global polynomial optimization problems by using sum of squares (SOS) methods or semidefinite programming (SDP) relaxations. The basic idea is to approximate a semialgebraic set $S$ by a collection of convex sets called SDP relaxations each of which has an SDP representation. This leads to the fundamental problem of which sets can be represented with LMIs or projections of LMIs.

A set $S$ is said to have an $L M I$ representation or be LMI representable if

$$
S=\left\{x \in \mathbb{R}^{n}: A_{0}+\sum_{i=1}^{n} A_{i} x_{i} \succeq 0\right\}
$$

for some symmetric matrices $A_{i}$. Here the notation $X \succeq 0(\succ 0)$ means the matrix $X$ is positive semidefinite (definite). Obvious necessary conditions for $S$ to be LMI representable are that $S$ must be convex and $S$ must have the form

$$
S=\left\{x \in \mathbb{R}^{n}: g_{1}(x) \geq 0, \cdots, g_{m}(x) \geq 0\right\}
$$

where $g_{i}(x)$ are multivariate polynomials; such are called basic closed semialgebraic sets.

It turns out that many convex sets are not LMI representable, see Helton and Vinnikov HV07. For instance, the convex set

$$
\left\{x \in \mathbb{R}^{2}: 1-\left(x_{1}^{4}+x_{2}^{4}\right) \geq 0\right\}
$$

does not admit an LMI representation. However, the set $S$ is the projection onto $x$-space of the set

$$
\begin{gathered}
\hat{S}:=\left\{(x, w) \in \mathbb{R}^{2} \times \mathbb{R}^{2}:\left[\begin{array}{cc}
1 & x_{2} \\
x_{2} & w_{2}
\end{array}\right] \succeq 0\right. \\
\left.\left[\begin{array}{cc}
1+w_{1} & w_{2} \\
w_{2} & 1-w_{1}
\end{array}\right] \succeq 0,\left[\begin{array}{cc}
1 & x_{1} \\
x_{1} & w_{1}
\end{array}\right] \succeq 0\right\}
\end{gathered}
$$

in $\mathbb{R}^{4}$ which is representable by an LMI.

More generally a set $S \subseteq \mathbb{R}^{n}$ is said to be semidefinite representable or SDP representable if $S$ can be described as

$$
\begin{aligned}
& S=\left\{x \in \mathbb{R}^{n}: \exists w \in \mathbb{R}^{M}\right. \text { s.t. } \\
&\left.A+\sum_{i=1}^{n} x_{i} B_{i}+\sum_{j=1}^{M} w_{j} C_{j} \succeq 0\right\} .
\end{aligned}
$$

Here $A, B_{i}, C_{j}$ are symmetric matrices of appropriate dimensions. Conceptually, one can think of $S$ as the projection into $\mathbb{R}^{n}$ of a set $\hat{S}$ in $\mathbb{R}^{(n+M)}$ having the LMI representation:

$$
\begin{aligned}
& \hat{S}=\{(x, w) \in \mathbb{R}^{(n+M)}: \\
&\left.A+\sum_{i=1}^{n} x_{i} B_{i}+\sum_{j=1}^{M} w_{j} C_{j} \succeq 0\right\} .
\end{aligned}
$$


The representation (1.3) is called a semidefinite representation or $S D P$ representation of the set $S$. We refer to the $w_{j}$ as auxillary variables.

The key use of an SDP representation is illustrated by optimizing a linear function $\ell^{T} x$ over $S$. Note that minimizing $\ell^{T} x$ over $S$ is equivalent to problem

$$
\min _{(x, y) \in \hat{S}} \ell^{T} x
$$

which is a conventional LMI, so can be attacked by standard toolboxes. Nesterov and Nemirovski ([NN94]) in their book which introduced LMIs and SDP gave collections of examples of SDP representable sets thereby leading to:

Question: Which convex sets $S$ are the projection of a set $\hat{S}$ having an LMI representation?

In $§ 4.3 .1$ of his excellent 2006 survey Nem06, Nemirovsky commented "this question seems to be completely open". Now much more is known and this paper describes both qualitative theory and SDP constructions.

Recently, Helton and Nie HN1, HN2] proved some sufficient conditions that guarantee the convex set $S$ is SDP representable. For instance, one sufficient condition is called the so-called sos-convexity or sosconcavity. A polynomial $f(x)$ is called sos-convex if its Hessian matrix $\nabla^{2} f(x)=W(x)^{T} W(x)$ for some matrix polynomials ( $W(x)$ is not necessarily square). A polynomial $g(x)$ is called sos-concave if $-g(x)$ is sos-convex. Helton and Nie HN1 proved the following theorem:

Theorem 1.1 ( $\mathrm{HN1})$. If every $g_{i}(x)$ is sos-concave, then $S$ is $S D P$ representable.

An explicit construction of one SDP representation of $S$ when every $g_{i}(x)$ is sos-concave will be given in Section 2 For general polynomials $g_{i}(x)$, the constructed SDP representation in Section 2 is usually very big. However, when polynomials $g_{i}(x)$ are sparse, the SDP representation can be reduced to have smaller sizes. This will be addressed in Section 3

There are also some sufficient conditions other than sos-concavity that guarantee the SDP representability. For instance, when the boundary of $S$ is positively curved, then $S$ is SDP representable. This will be discussed in Section 4

\section{General SDP representation}

Suppose $S$ is a convex set given in the form

$$
S=\left\{x \in \mathbb{R}^{n}: g_{1}(x) \geq 0, \cdots, g_{m}(x) \geq 0\right\} .
$$

In this section, we assume every $g_{i}(x)$ is a sos-concave polynomial. A natural SDP relaxation of $S$ is

$$
R=\left\{x: \exists y \text { s.t. } g(x, y) \geq 0, M_{d}(x, y) \succeq 0\right\} .
$$

Here $g(x, y)$ is a vector valued linear function and $M_{d}(x, y)$ is a matrix valued linear function defined in what follows. The integer $2 d$ is the minimum upper bound of the degrees of $g_{i}(x)$. The vector $g(x, y)$ is of the form

$$
g(x, y)=\tilde{g}_{0}+\sum_{i=1}^{n} x_{i} \tilde{g}_{i}+\sum_{1<|\alpha| \leq 2 d} y_{\alpha} \tilde{g}_{\alpha}
$$

whose coefficients are such that

$$
\left[\begin{array}{c}
g_{1}(x) \\
\vdots \\
g_{m}(x)
\end{array}\right]=\tilde{g}_{0}+\sum_{i=1}^{n} x_{i} \tilde{g}_{i}+\sum_{1<|\alpha| \leq 2 d} x^{\alpha} \tilde{g}_{\alpha}
$$

The matrix $M_{d}(x, y)$ is the $d$-th order moment matrix constructed as

$$
M_{d}(x, y)=A_{0}+\sum_{i=1}^{n} x_{i} A_{i}+\sum_{1<|\alpha| \leq 2 N} y_{\alpha} A_{\alpha} .
$$

Here the symmetric matrices $A_{\alpha}$ are such that

$$
\mathbf{m}_{d}(x) \mathbf{m}_{d}(x)^{T}=A_{0}+\sum_{i=1}^{n} x_{i} A_{i}+\sum_{1<|\alpha| \leq 2 d} x^{\alpha} A_{\alpha} .
$$

The notation $\mathbf{m}_{d}(x)$ above denotes the column vector of monomials with degree up to $d$, i.e.,

$$
\mathbf{m}_{d}(x)=\left[\begin{array}{lllllll}
1 & x_{1} & \cdots & x_{1}^{2} & x_{1} x_{2} & \cdots & x_{n}^{d}
\end{array}\right]^{T} .
$$

This construction of SDP relaxations of the set $S$ was proposed by Parrilo Par06. and Lasserre Las06. When every $g_{i}(x)$ is sos-concave, Helton and Nie [HN1] proved $R=S$. This result lends itself to implementation which we now illustrate with two examples. After that we improve this SDP construction to exploit sparsity structure when it is present in the defining polynomials $g_{i}$.

Example 2.1. Consider the set $S=\left\{x \in \mathbb{R}^{n}\right.$ : $g(x) \geq 0\}$ where

$$
g(x)=1-\left(x_{1}^{4}+x_{2}^{4}-x_{1}^{2} x_{2}^{2}\right) .
$$

Direct calculation shows

$$
-\nabla^{2} g(x)=\left[\begin{array}{ll}
x_{1} & \\
& x_{2}
\end{array}\right] \underbrace{\left[\begin{array}{cc}
12 & -4 \\
-4 & 12
\end{array}\right]}_{\succeq 0}\left[\begin{array}{ll}
x_{1} & \\
& x_{2}
\end{array}\right] .
$$


So $g(x)$ is sos-concave. Thus we know $S$ can be represented by $R$ constructed in (2.4), which in this specification becomes

$$
\left[\begin{array}{cccccc}
1 & x_{1} & x_{2} & y_{20} & y_{11} & y_{02} \\
x_{1} & y_{20} & y_{11} & y_{30} & y_{21} & y_{12} \\
x_{2} & y_{11} & y_{03} & y_{21} & y_{12} & y_{03} \\
y_{20} & y_{30} & y_{21} & y_{40} & y_{31} & y_{22} \\
y_{11} & y_{21} & y_{12} & y_{31} & y_{22} & y_{13} \\
y_{02} & y_{12} & y_{03} & y_{22} & y_{13} & y_{04}
\end{array}\right] \succeq 0 .
$$

The matrix above is the second order moment matrix. In this SDP representation, there are 12 auxiliary variables $y_{i j}$.

Example 2.2. Consider the set $S=\left\{x \in \mathbb{R}^{n}: 1-\right.$ $p(x) \geq 0\}$ where $p$ is a homogeneous polynomial:

$$
p(x)=\left[x^{d}\right]^{T} B\left[x^{d}\right] .
$$

Here $d>0$ is an integer and

$$
B=\left(b_{i j}\right)_{1 \leq i, j \leq n} \succeq 0
$$

is a symmetric matrix, and $\left[x^{d}\right]$ denotes the vector

$$
\left[x^{d}\right]=\left[\begin{array}{llll}
x_{1}^{d} & x_{2}^{d} & \cdots & x_{n}^{d}
\end{array}\right]^{T} .
$$

Direct calculation shows

$$
\nabla^{2} p(x)=\operatorname{diag}\left(\left[x^{d-1}\right]\right) \cdot W \cdot \operatorname{diag}\left(\left[x^{d-1}\right]\right)
$$

where the symmetric matrix $W$ is defined to be

$$
W=d^{2} B+\left(3 d^{2}-2 d\right) \operatorname{diag}(B) .
$$

Since $B \succeq 0$, we also have $W \succeq 0$. So $p(x)$ is sosconvex. Therefore $S$ can be represented as

$$
\left\{x: \exists y, 1-\sum_{i, j=1}^{n} b_{i j} y_{d\left(e_{i}+e_{j}\right)} \geq 0, M_{d}(x, y) \succeq 0\right\}
$$

Here $e_{i}$ denotes the $i$-th standard unit basis vector of $\mathbb{R}^{n}$ and $M_{d}(x, y)$ is the $d$-th order moment matrix.

\section{Sparse SDP representation}

The SDP relaxations in Las06, HN1, HN2, Par06 have not exploited the special structures of polynomials

$$
g_{1}(x), \cdots, g_{m}(x)
$$

such as dependence of each polynomial on only a few variables (termed sparsity). On the other hand, in polynomial optimization the sparsity structure of polynomials can be exploited to improve the computation efficiency of their semidefinite relaxations KKW05, Las06spr, Nie06, ND06, Par03, WKKM06]. In this paper we show that when the defining polynomials for $S$ are sparse, their structures can also be exploited to get a "sparser" SDP representation.

This section gives a structured SDP relaxation and proves a sufficient condition such that this structured SDP relaxation represents $S$ exactly.

Throughout this section, we assume every polynomial $g_{k}(x)$ is sos-concave. Let

$$
\mathcal{H}=\left\{x \in \mathbb{R}^{n}: a^{T} x \geq b\right\} \supseteq S
$$

be a supporting half space and $a^{T} u=b$ for some $u \in \partial S$. When $S$ has nonempty interior, there exists Lagrange multipliers $\lambda_{1} \geq 0, \cdots, \lambda_{m} \geq 0$ such that

$$
a=\sum_{k=1}^{m} \lambda_{k} \nabla g_{k}(u), \lambda_{i} g_{i}(u)=0, i=1, \cdots, m .
$$

Helton and Nie HN1 showed that the Lagrange function

$$
a^{T} x-b-\sum_{k=1}^{m} \lambda_{k} g_{k}(x)
$$

is an SOS polynomial when every polynomial $g_{k}(x)$ is sos-concave.

Now we suppose the polynomials $g_{k}(x)$ are structured such that, for any $a, b, \lambda$, there is a decomposition such that

$a^{T} x-b-\sum_{k=1}^{m} \lambda_{k} g_{k}(x)=\phi_{\lambda}^{(1)}\left(x_{I_{1}}\right)+\cdots+\phi_{\lambda}^{(K)}\left(x_{I_{K}}\right)$,

where each $\phi_{\lambda}^{(i)}\left(x_{I_{i}}\right)$ is a polynomial in variables $x_{I_{i}}$. $\left\{I_{1}, \cdots, I_{K}\right\}$ is a partition of the index set $\{1,2, \cdots, n\}$ such that $I_{i} \cap I_{j}=\emptyset$ whenever $i \neq j$. $x_{I_{i}}$ denotes the subvector of $x$ whose indices are in $I_{i}$. In other words, the polynomials

$$
\phi_{\lambda}^{(1)}\left(x_{I_{1}}\right), \cdots, \phi_{\lambda}^{(K)}\left(x_{I_{K}}\right)
$$

are uncoupled.

Given a polynomial $p(x)$, denote by $\operatorname{supp}(p(x))$ the support of $p(x)$, i.e., the set of exponents of existing monomials of $p(x)$. If $p(x)$ is SOS and has decomposition $p(x)=\sum_{i} q_{i}^{2}(x)$, then it holds

$$
\operatorname{supp}\left(q_{i}(x)\right) \subseteq \text { convex hull }\left(\frac{1}{2} \operatorname{supp}(p(x))\right),
$$

by Theorem 1 in Reznick Rez78. So we define $F_{i}$ to be the maximum lattice set such that

$$
F_{i} \subseteq \text { convex hull }\left(\frac{1}{2} \operatorname{supp}\left(\phi_{\lambda}^{(i)}\right)\right) .
$$


Now define symmetric matrices $M_{\alpha}^{j}$ as follows

$$
\begin{gathered}
\mathbf{m}_{F_{i}}\left(x_{I_{i}}\right) \mathbf{m}_{F_{i}}\left(x_{I_{i}}\right)^{T} \\
=M_{0}^{(i)}+\sum_{j \in I_{i}} x_{j} M_{j}^{(i)}+\sum_{1<|\alpha| \leq 2 N} x^{\alpha} M_{\alpha}^{(i)} .
\end{gathered}
$$

Here $\mathbf{m}_{F_{i}}\left(x_{I_{i}}\right)$ denotes the vector of monomials whose exponents lie in $F_{i}$. Then define linear matrices

$$
M_{F_{i}}(x, y)=M_{0}^{(i)}+\sum_{j \in I_{i}} x_{j} M_{j}^{(i)}+\sum_{1<|\alpha| \leq 2 d} x^{\alpha} M_{\alpha}^{(i)} .
$$

Lemma 3.1. Let $a, b, \lambda$ be the above. Then there are symmetric matrices $W_{1}, \cdots, W_{K} \succeq 0$ such that

$$
a^{T} x-b-\sum_{k=1}^{m} \lambda_{k} g_{k}(x)=\sum_{i=1}^{K} \mathbf{m}_{F_{i}}\left(x_{I_{i}}\right)^{T} \cdot W_{i} \cdot \mathbf{m}_{F_{i}}\left(x_{I_{i}}\right) .
$$

Proof. By the structure assumption, we have representation

$$
\begin{aligned}
L_{a}(x) & :=a^{T} x-b-\sum_{k=1}^{m} \lambda_{k} g_{k}(x) \\
& =\eta_{1}\left(x_{I_{1}}\right)+\cdots+\eta_{K}\left(x_{I_{K}}\right)
\end{aligned}
$$

for some polynomials $\eta_{1}\left(x_{I_{1}}\right), \cdots, \eta_{K}\left(x_{I_{K}}\right)$. We know $L_{a}(x)$ is nonnegative polynomial and $u$ is one global minimizer such that $L_{a}(u)=0$. Let $u^{(i)}$ denote the subvector of $u$ whose coordinates correspond to the variables $x_{I_{i}}$. Then $u^{(i)}$ is one global minimizer of $\eta_{i}\left(x_{I_{i}}\right)$. So we know

$$
L_{a}(x)=\sum_{i=1}^{k}\left(\eta_{i}\left(x_{I_{i}}\right)-\eta_{i}\left(u^{(i)}\right)\right)
$$

is SOS by Section 3 in HN1. In the above, fix one index $i$ and set $x^{(j)}=u^{(j)}$ for $j \neq i$, then we can see $\eta_{i}\left(x_{I_{i}}\right)-\eta_{i}\left(u^{(i)}\right)$ must also be SOS in $x_{I_{i}}$. Furthermore, by Theorem 1 in Reznick Rez78, the polynomial $\eta_{i}\left(x_{I_{i}}\right)-\eta_{i}\left(u^{(i)}\right)$ has the representation

$$
\eta_{i}\left(x_{I_{i}}\right)-\eta_{i}\left(u^{(i)}\right)=\mathbf{m}_{F_{i}}\left(x_{I_{i}}\right)^{T} \cdot W_{i} \cdot \mathbf{m}_{F_{i}}\left(x_{I_{i}}\right),
$$

for some symmetric matrix $W_{i} \succeq 0$. Thus the Lemma is proven.

Theorem 3.2. Under the above assumptions, the convex set $S$ has the $S D P$ representation

$$
\begin{aligned}
L=\left\{x \in \mathbb{R}^{n}: \exists y, \text { s.t. } g(x, y) \geq 0,\right. \\
\\
\left.\quad M_{F_{i}}(x, y) \succeq 0, i=1, \cdots, K\right\} .
\end{aligned}
$$

That is, $S=L$.
Proof. We have seen $S \subseteq L$. If $L \neq S$, then there must exist some point $\hat{x} \in L / S$. By the Convex Set Separation Theorem, there exists one supporting hyperplane of $S$

$$
\mathcal{H}=\left\{x \in \mathbb{R}^{n}: a^{T} x \geq b\right\} \supseteq S
$$

such that $a^{T} u=b$ for some $u \in \partial S$ and $a^{T} \hat{x}<b$. Consider the linear optimization problem

$$
\begin{aligned}
b=\min _{x \in \mathbb{R}^{n}} & a^{T} x \\
\text { s.t. } & g_{1}(x) \geq 0, \cdots, g_{m}(x) \geq 0 .
\end{aligned}
$$

Then $u$ is one minimizer for the above. Let $\lambda_{1} \geq$ $0, \cdots, \lambda_{m} \geq 0$ be the corresponding Lagrange multipliers. Then, by the previous lemma, we have shown

$$
\begin{gathered}
a^{T} x-b-\sum_{i=1}^{m} \lambda_{k} g_{i}(x) \\
=\sum_{i=1}^{K} \mathbf{m}_{F_{i}}\left(x_{I_{i}}\right)^{T} \cdot W_{i} \cdot \mathbf{m}_{F_{i}}\left(x_{I_{i}}\right)
\end{gathered}
$$

for some symmetric matrices $W_{1}, \cdots, W_{K} \succeq 0$. So we have

$$
\begin{gathered}
b=\max \gamma \text { s.t. } \\
a^{T} x-\gamma-\sum_{i=1}^{m} \lambda_{k} g_{i}(x)=\sum_{i=1}^{K} \mathbf{m}_{F_{i}}\left(x_{I_{i}}\right)^{T} \cdot W_{i} \cdot \mathbf{m}_{F_{i}}\left(x_{I_{i}}\right) \\
\lambda_{1}, \cdots, \lambda_{m} \geq 0, W_{1}, \cdots, W_{K} \succeq 0 .
\end{gathered}
$$

The dual of the above SOS program is

$$
\min a^{T} x \quad \text { s.t. } x \in L .
$$

Since $\hat{x} \in L$, by weak duality, it holds $b \leq a^{T} \hat{x}$, which contradicts the previous assertion $a^{T} \hat{x}<b$.

Now let us show some examples for the sparse SDP representation constructed in (3.7).

Example 3.3. Consider the convex set

$S=\left\{x \in \mathbb{R}_{+}^{n}: g(x):=1-\left(x_{1}^{8}+x_{1}^{2}+x_{1} x_{2}+x_{2}^{2}\right) \geq 0\right\}$.

Obviously $g(x)$ is sos-concave. The convex hull of $\left(\frac{1}{2} \operatorname{supp}(g)\right)$ contains only the following integer points:

$$
(0,0),(1,0),(2,0),(3,0),(4,0),(0,1) .
$$

By the sparsity theorem, $S$ can be represented as

$$
\left[\begin{array}{cccccc}
1-y_{80}-y_{20}-y_{11}-y_{02} \geq 0, \\
x_{1} & x_{1} & x_{2} & y_{20} & y_{30} & y_{40} \\
x_{2} & y_{11} & y_{02} & y_{21} & y_{40} & y_{50} \\
y_{20} & y_{30} & y_{21} & y_{40} & y_{50} & y_{60} \\
y_{30} & y_{40} & y_{31} & y_{50} & y_{60} & y_{70} \\
y_{40} & y_{50} & y_{41} & y_{60} & y_{70} & y_{80}
\end{array}\right] \succeq 0 .
$$

The matrix above is the sparse moment matrix constructed in (3.6). There are totally 11 auxiliary variables $y_{i j}$. 
Example 3.4. Consider the set $S=\left\{x \in \mathbb{R}^{n}: 1-\right.$ $p(x) \geq 0\}$ where

$$
p(x)=\sum_{i=1}^{n} p_{i}\left(x_{i}\right), \quad p_{i}\left(x_{i}\right)=\sum_{k=1}^{2 d} \frac{x_{i}^{k}}{k !} .
$$

Obviously $p(x)$ is sos-convex, because each univariate polynomial $p_{i}\left(x_{i}\right)$ is convex and hence sos-convex. Thus $S$ can be represented as

$$
\begin{gathered}
1-\sum_{i=1}^{n} \sum_{k=1}^{2 d} \frac{y_{k}^{(i)}}{k !} \geq 0 \\
H_{1}\left(x_{1}, y^{(i)}\right) \succeq 0, \cdots, H_{n}\left(x_{n}, y^{(n)}\right) \succeq 0
\end{gathered}
$$

where $H_{i}\left(x_{i}, y^{(i)}\right)$ are defined as

$$
H_{i}\left(x_{i}, y^{(i)}\right)=\left[\begin{array}{ccccc}
1 & x_{i} & y_{2}^{(i)} & \cdots & y_{d}^{(i)} \\
x_{i} & y_{2}^{(i)} & y_{3}^{(i)} & \cdots & y_{d+1}^{(i)} \\
y_{2}^{(i)} & y_{3}^{(i)} & y_{4}^{(i)} & \cdots & y_{d+2}^{(i)} \\
\vdots & \vdots & \vdots & \ddots & \vdots \\
y_{d}^{(i)} & y_{d+1}^{(i)} & y_{d+2}^{(i)} & \cdots & y_{2 d}^{(i)}
\end{array}\right] .
$$

The symmetric matrices $H_{i}\left(x_{i}, y^{(i)}\right)$ are sparse moment matrices constructed in (3.6). There are totally $2 n(d-1)$ auxiliary variables $y_{k}^{(i)}$.

\section{Positive curvature condition}

Section 2 and Section 3 show the explicit construction of SDP representation when all the defining polynomials $g_{i}(x)$ are sos-concave. If some $g_{i}(x)$ is not sosconcave, these constructions usually do not represent $S$. However, there are other sufficient conditions that guarantees $S$ is SDP representable, which is called positive curvature.

Assume $S$ in (1.2) is convex, compact and has nonempty interior. Denote by $\partial S$ the boundary of $S$. Let $Z_{i}=\left\{x: g_{i}(x)=0\right\}$ and note $\partial S \subset \cup_{i} Z_{i}$. We say the defining functions of $S$ are nondegenerate provided $\nabla g_{i}(x) \neq 0$ for all $x \in Z_{i} \cap \partial S$. The boundary of $S$ is said to have positive curvature provided that there exist nondegenerate defining functions $g_{i}$ for $S$ such that at each $x \in \partial S \cap Z_{i}$

$$
-v^{T} \nabla^{2} g_{i}(x) v>0, \quad \forall 0 \neq v \in \nabla g_{i}(x)^{\perp},
$$

in other words, the Hessian of $g_{i}$ compressed to the tangent space (the second fundamental form) is negative definite. A standard fact in geometry is that this does not depend on the choice of $g_{i}(X)$.

Obviously, necessary conditions for $S$ to be SDP representable are that $S$ must be convex and semialgebraic (describable by a system of polynomial equalities or inequalities over the real numbers). The following, Theorem 3.3 of HN2, goes in the direction of the converse.
Theorem 4.1. Suppose $S$ is a convex compact set with nonempty interior which has nondegenerate defining polynomials $S=\left\{x \in \mathbb{R}^{n}: g_{1}(x) \geq 0, \cdots, g_{m}(x) \geq\right.$ $0\}$. If the boundary $\partial S$ is positively curved, then $S$ is SDP representable.

If $S$ is convex with nondegenerate defining functions, then its boundary has nonnegative curvature. Thus the positive curvature assumption is not a huge restriction beyond being strictly convex. The nondegeneracy assumption is another restriction.

Finally comes an example where the defining polynomial is not concave but the boundary has positive curvature.

Example 4.2. Consider the set

$$
S=\left\{x \in \mathbb{R}_{+}^{n}: g(x):=x_{1} x_{2} \cdots x_{n}-1 \geq 0\right\} .
$$

We can easily see that $S$ is convex but the defining polynomial $g(x)$ is not concave. Note that

$$
\begin{gathered}
\frac{\nabla g(x)}{g(x)+1}=\left[\begin{array}{llll}
\frac{1}{x_{1}} & \frac{1}{x_{2}} & \cdots & \frac{1}{x_{n}}
\end{array}\right]^{T} \\
\frac{\nabla^{2} g(x)}{g(x)+1}=\left[\begin{array}{cccc}
0 & \frac{1}{x_{1} x_{2}} & \cdots & \frac{1}{x_{1} x_{n}} \\
\frac{1}{x_{1} x_{2}} & 0 & \cdots & \frac{1}{x_{2} x_{n}} \\
\vdots & \vdots & \ddots & \vdots \\
\frac{1}{x_{1} x_{n}} & \cdots & \frac{1}{x_{n-1} x_{n}} & 0
\end{array}\right] .
\end{gathered}
$$

We claim that the boundary $\partial S$ has positive curvature, which is justified by the following observation:

$$
\begin{gathered}
-\nabla^{2} g(x)+\nabla g(x) \nabla g(x)^{T} \\
\succeq(g(x)+1) \operatorname{diag}\left(\frac{1}{x_{1}^{2}}, \frac{1}{x_{2}^{2}}, \cdots, \frac{1}{x_{n}^{2}}\right) \succ 0, \forall x \in \partial S .
\end{gathered}
$$

Since $\partial S$ has positive curvature, Theorem 4.1 guarantees $S$ has an SDP representation whose construction was in Section 5 in HN1.

\section{Concluding remarks}

This paper gives an explicit construction, (2.4), of an SDP representation for a convex set $S$ and a sparser one (3.7) when polynomials $g_{k}(x)$ are sos-concave. There are also some other constructions of SDP relaxations Las06, HN1, HN2 for $S$, which are also SDP representations of $S$ when $g_{k}(x)$ are strictly concave on the boundary $\partial S$ of $S$ or when the boundary $\partial S$ has positive curvature.

In theory a hierarchy of SDP relaxations converging to $S$ within finitely many steps can be constructed when the boundary $\partial S$ has positive curvature (weaker than our hypothesis). However, these refined constructions of SDP representations are usually more complicated than (2.4) or (3.7), for example, usually 
it is difficult to predict which step of their hierarchy of relaxations represents $S$ exactly. In contrast, the size of construction (2.4) or (3.7) is explicit. We refer to $\mathrm{HN1}$, HN2 for more details.

\section{References}

[HN1] J.W. Helton and J. Nie. Semidefinite Representation of Convex Sets. Preprint, 2007. http://arxiv.org/abs/0705.4068.

[HN2] J.W. Helton and J. Nie. Sufficient and Necessary Conditions for Semidefinite Representability of Convex Hulls and Sets. Preprint, 2007. http://arxiv.org/abs/0709.4017

[HV07] Helton, J. W. and Vinnikov, V., Linear matrix inequality representation of sets, Communications On Pure \& Appl Math, 60, p654-674, 5, 2007

[KKW05] M. Kojima, S. Kim and H. Waki. Sparsity in Sums of Squares of Polynomials. Math. Prog., Vol. 103 (1), 45-62 (2005).

[Las01] J. B. Lasserre. Global optimization with polynomials and the problem of moments. SIAM J. Optim., 11(3): 796-817, 2001.

[Las06spr] J. Lasserre. Convergent SDP-relaxations in polynomial optimization with sparsity. SIAM J. on Optim., Vol. 17, No. 3, pp.822-843, 2006.

[Las06] J. Lasserre. Convex sets with lifted semidefinite representation. Preprint, LAAS, 2006.

[Nem06] A. Nemirovskii. Advances in convex optimization: conic programming. Plenary Lecture, International Congress of Mathematicians (ICM), Madrid, Spain, 2006.

[NN94] Y. Nesterov and A. Nemirovskii. Interiorpoint polynomial algorithms in convex programming. SIAM Studies in Applied Mathematics, 13. Society for Industrial and Applied Mathematics (SIAM), Philadelphia, PA, 1994.

[Nie06] J. Nie. Sum of squares method for sensor network localization. To appear in Computational Optimization and Applications.

[NDS06] J. Nie, J. Demmel and B. Sturmfels. Minimizing polynomials via sum of squares over the gradient ideal. Math. Prog., Series A, Vol. 106 (2006), No. 3, pp. 587-606.

[ND05] J. Nie and J. Demmel. Minimum ellipsoid bounds for solutions of polynomial systems via sum of squares. Journal of Global Optimization, Vol. 33(2005), pp. 511-525.
[ND06] J. Nie and J. Demmel. Sparse SOS relaxations for minimizing functions that are summations of small polynomials. Preprint, 2006. http://arxiv.org/abs/math/0606476

[NPS08] J. Nie, P. Parrilo and B. Sturmfels. Semidefinite Representation of the k-Ellipse. IMA Volume 146: Algorithms in Algebraic Geometry (Eds. A. Dickenstein, F.-O. Schreyer, and A. Sommese), Springer, New York, 2008.

[Par00] P. Parrilo. Structured semidefinite programs and semialgebraic geometry methods in robustness and optimization. $\mathrm{PhD}$ thesis, California Institute of Technology, May 2000.

[ParStu03] P. A. Parrilo and B. Stunnfels. Minimizing polynomial functions. In S. Basu and L. Gonzalez-Vega, editors, Algorithmic and Quantitative Aspects of Real Algebraic Geometry in Mathematics and Computer Science, volume 60 of DIMACS Series in Discrete Mathematics and Computer Science, pages 83-99. AMS, 2003.

[Par03] P.A. Parrilo. Exploiting structure in sum of squares programs. Proceedings for the 42nd IEEE Conference on Decision and Control, Maui, Hawaii, 2003.

[Par06] P.Parrilo. Exact semidefinite representation for genus zero curves. Talk at the Banff workshop "Positive Polynomials and Optimization", Banff, Canada, October 8-12, 2006.

[Rez78] B. Reznick. Extremal psd forms with few terms. Duke Math. J., 45(1978), No. 2, pp. 363374.

[WKKM06] H. Waki, S. Kim, M. Kojima and M. Muramatsu. Sums of Squares and Semidefinite Programming Relaxations for Polynomial Optimization Problems with Structured Sparsity. SIAM J. on Optim., Vol. 17 No. 1 pp. 218-242 (2006). 